\title{
Methodology to Calculate Boundary Conditions in a Single Isolated Helically Segmented Finned Tube Module
}

\author{
E. Martínez, W. Vicente, G. Soto, A. Campo and M. Salinas \\ Additional information is available at the end of the chapter
}

http://dx.doi.org/10.5772/51995

\section{Introduction}

Helically segmented finned tubes are used in compact heat recoveries in order to save energy in industrial applications. These equipments are small because the gas phase turbulence and the heat transfer surface are increased by the presence of fins; both are relevant in heat transfer. However, the gas phase pressure drop is elevated and consequently, operational problems such as backpressure can emerge. Therefore, a study focusing on finned tubes is important in order to understand the fluid dynamics and heat transfer phenomena. There are two main methods for the analysis; the first uses integral analysis (gross effects) and the second uses Computational Fluid Dynamics (CFD) techniques. Integral analyses allow a quick evaluation of thermo-physical phenomena with minimum computational infrastructure but only gross effects can be examined. These analyses are primarily used in the design of equipment because only inlet and outlet fluid properties are important. The CFD technique requires good computational support and long calculation times, but it provides complete and detailed information on the intricate thermophysical phenomena. This modern analysis requires a correct implementation of boundary conditions in order to adequately represent the flow hydrodynamics and heat transfer phenomena.

The implementation of boundary conditions is relevant in differential analyses because predictions depend from it. The differential analyses can be developed by means of analytical solutions or by means of numerical methods. In the case of helically segmented finned tube bank analyses, the analytical solutions are not possible because the geometry is complex. Then, a numerical simulation of helically segmented finned tube bank is the best option. The numerical simulations can be carried out by means of three different CFD 
alternatives such as Direct Numerical Simulation (DNS), Large Eddy Simulation (LES), and Reynolds Average Navier-Stokes Equations (RANS). The DNS technique is limited to low Reynolds flows with simple configurations. The LES technique is less demanding than the DNS, but it takes considerable computing resources and computing time because the required calculations are always three-dimensional and unsteady. Finally, the RANS technique, which is widely used in industrial applications, considers average spatial and temporal scales of turbulent fluctuations and solves the transport equations as a function of these average variables. However, these equations are not closed and additional models (turbulence models) are indispensable to close the system. So, the numerical analysis on helically segmented finned tube bank (complex geometry) is proposed with the RANS technique.

In the open literature there are several papers have been focused on numerical analysis of small finned tube banks, the majority of them are restricted to numerical simulations on bare tube layout (symmetric tube layout). For example, Beale and Spalding [1], Comini and Croce [2] and Beale [3, 4] have performed simulations on laminar flow regimes exclusively. Other authors like Benhamadouche and Laurennce [5] and Salinas-Vazquez et al. [6] have conducted studies on turbulent flow regimes. These simulations have analyzed symmetric tube layout with periodic boundary conditions. On the other hand, there are few numerical simulations focused on helically segmented finned tubes (asymmetric finned tubes). The papers are focused on laminar flows with Dirichlet boundary conditions. For example, Hofmann [7] and Mcilwain [8,9] conducted two-dimensional simulations on a single helically segmented finned tube. Afterwards, Lemouedda et al. [10] developed a three-dimensional numerical simulation in a small finned tube bank. There are no reports of numerical simulations of asymmetric finned tube layout (helically segmented finned tube bank) under periodic boundary conditions and the effect of inside fluid temperature has not been considered. Therefore, a methodology to calculate flow properties in different zones of finned tube bank is required in order to implement boundary conditions on a single isolated finned tube module. This methodology considers calculations in entire and partial finned tube layout and it is applied to calculate boundary conditions in a numerical simulation. Then, a compact heat recovery in staggered layout is represented as some single isolated finned tube modules in order to save computational resources. The single isolated finned tube module is simulated and predictions are compared with results from correlations available in the open literature.

\section{Methodology}

The differential analysis of compact heat recoveries with CFD techniques requires high calculation times because a full finned tube bank needs to be simulated. The dimensions of computational domain are high due to size of finned tubes and number of finned tubes used in the equipment. So, the necessity to reduce calculation times is relevant in numerical simulations because these times can be excessively-high. The only way to reduce calculation times is by reduction of computational domain but it requires a correct implementation of boundary conditions for representing adequately physical phenomena. A complete finned 
tube bank can be represented by a single isolated finned tube module in the fully developed flow region as shown in figure 1. The single isolated finned tube module consists of an arrangement of entire and partial finned tubes. This finned tube layout can allow a computational domain reduction of $99 \%$ but requires values of boundary conditions in intermediate regions of finned tube bank. The values of boundary conditions for velocity, pressure, and temperature should be calculated for entire and partial finned tubes in intermediate regions of finned tube bank. Then, a method to calculate those boundary conditions must be developed in order to represent the finned tube bank as a single isolated finned tube module (figure 1). This methodology is based on integral models which have been validated experimentally [22] with precision higher than $90 \%$ for pressure and $95 \%$ for temperature. So, a numerical simulation of single isolated finned tube module in the fully developed flow with periodic boundary conditions is done. Numerical predictions are compared with results obtained from best correlations available in the open literature, which are presented in section 2.2. So, the mean pressure drop, mean temperature difference, mean Nusselt number and mean friction factor are compared.

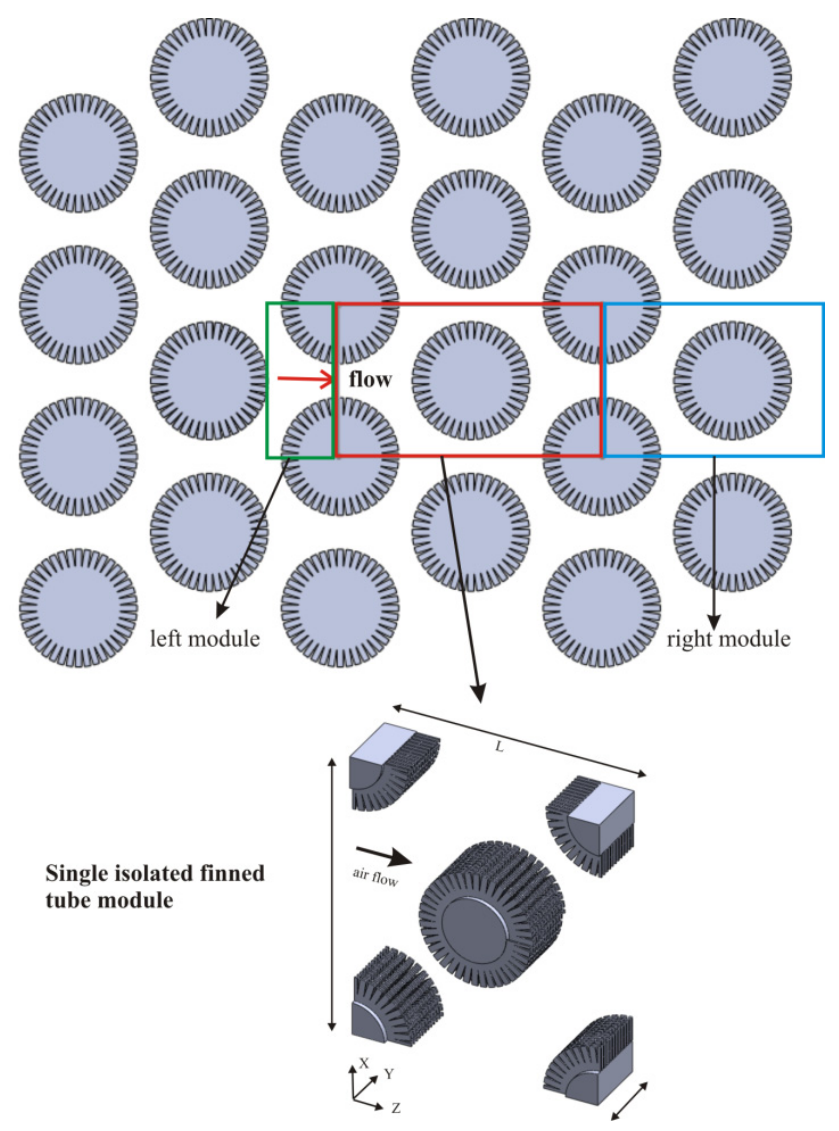

Figure 1. Finned tube bank in staggered layout. 


\subsection{Finned tube bank analysis}

The complete finned tube bank may be represented as a single isolated fined tube module in the fully developed flow as shown in figure 1 . This finned tube layout requires values for velocity, pressure, and temperature in intermediate regions of finned tube layout for both outside and inside flows. These values are obtained by means of an integral analysis, which considers the Logarithmic Mean Temperature Difference method (LMTD). However, this method only can be applied for entire finned tubes in arrangement of two or more finned tube rows and two or more finned tubes per row. Then, the complete finned tube bank and the single isolated finned tube module should be analyzed in order to apply LMTD method, which is described in section 2.2. The finned tube bank can be divided in single isolated finned tube modules as shown in figure 2a. In this figure, a full finned tube bank (6 finned tube rows with 4 finned tubes per row) is composed by 12 single isolated finned tube modules (black and red boxes). Every finned tube module contains 2 finned tubes because there are one entire finned tube and 4 quarters of finned tube. Then, two single isolated finned tube modules like (red boxes) can be represented as an equivalent small finned tube bank (blue box) as shown in figure $2 \mathrm{~b}$. Therefore, the finned tube bank showed in figure 1 is represented as a single isolated finned tube modules arrangement as presented in figure $2 \mathrm{a}$.

Figure $2 \mathrm{~b}$ shows a small finned tube bank (blue box) composed of 2 finned tubes per row and 2 rows of finned tubes, which is arranged in order to obtain 2 single isolated finned tube modules (red box). Then, the analysis of single isolated finned tube modules must consider minimum arrangements of 2 finned tubes per row and 2 rows of finned tubes. This consideration does not affect predictions of friction factor and Nusselt number because these dimensionless parameters are not function of number of finned tubes involved in the arrangement if mass flow is corrected to the new finned tube layout, which is demonstrated in the sensitive analysis (section 4.1). So, models for evaluating heat transfer and pressure drop can be applied to the equivalent small finned tube bank (figure $2 b$ ). The pressure drop depends mainly of flow hydrodynamics, which shows similar velocity fields for every single isolated finned tube module as is discussed in results analysis (section 4.2). Therefore, the models for evaluating pressure drop can be applied directly in the equivalence small finned tube bank (figure $2 \mathrm{~b}$ ). However, the pressure drop cannot be considered as a boundary condition because only represents the pressure difference at the inlet and exit of single isolated finned tube modules. The pressure in the boundaries of single isolated finned tube module is obtained with the analysis of finned tube bank from figure 1 . In this figure, the single isolated finned tube in the fully developed region (red box) is located near to the exit of module. This finned tube module has to the right a part of finned tube module (blue box) while at left has one and a part finned tube module (green box). In the case of an atmospheric discharge of flow gases (zero relative pressure), which is correct because flue gases of compact heat recoveries cannot be used in additional industrial process, the relative pressure drop in the last part of single isolated finned tube module (blue box, figure 1) is calculated as an proportional arithmetic mean pressure drop to the part of this finned tube module. The proportional part of this module corresponds to a value of 0.75 . Therefore, the relative pressure at the exit of the single isolated finned tube module in the fully developed 
flow (red box, figure 1) corresponds to the value calculated previously. Finally, the relative pressure at the inlet of the single isolated finned module (red box, figure 1) is calculated from the sum of the pressure drop in this finned tube module and the proportional part (0.25) of the left finned tube module (green box, figure 1).

a)

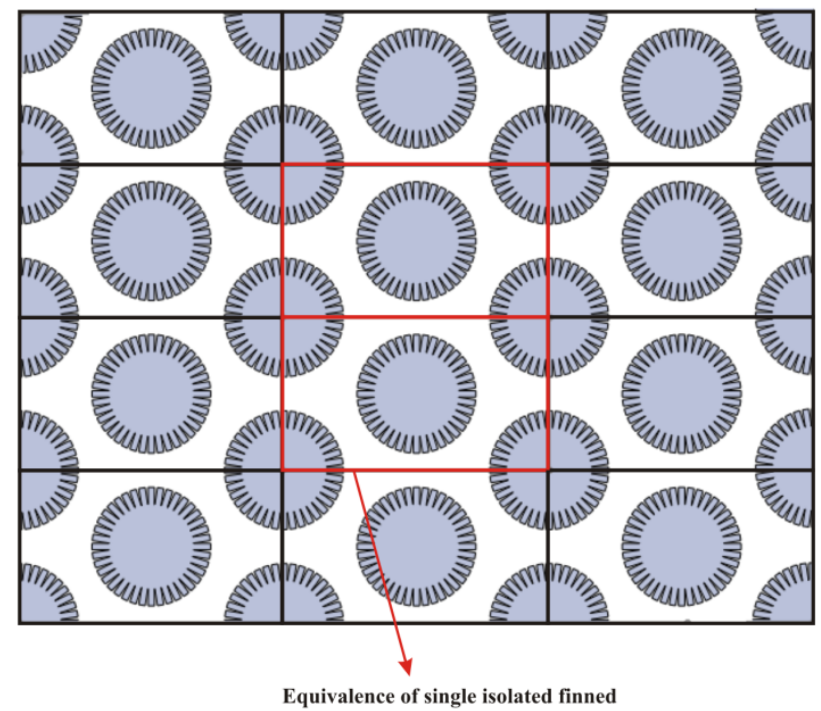

b)

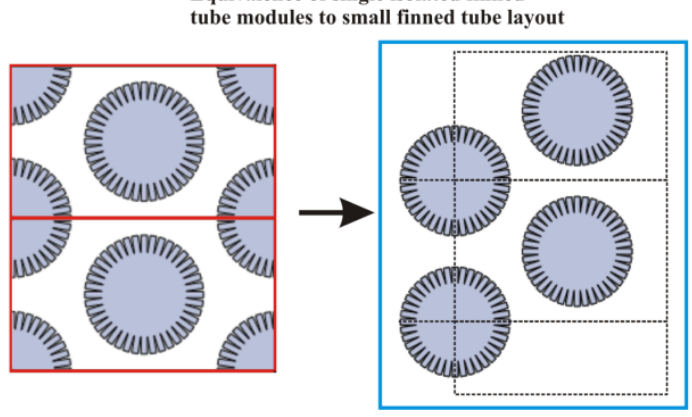

Figure 2. Single isolated finned tube modules in the finned tube bank.

The direct application of LMDT method for heat transfer in the equivalent small finned tube bank (blue box, figure $2 \mathrm{~b}$ ) is not recommended because the outside flow, in the single isolated finned tube module (red box, figure $2 b$ ), is cooled by tree different cooling sources (inside fluid temperature). While the equivalent small finned tube bank (blue box, figure 2) only has the influence of two cooling sources. One way to solve this problem is by means of temperature evaluation of outside flow for small finned tube layouts. The finned tube layouts considered have an initial arrangement of two finned tube rows, which are evaluated. Later, calculations over initial finned tube bank with additional finned tube rows are proposed (see section 2.3). This procedure is iterative because only initial conditions at the inlet of gas-phase flow and inside fluid in the complete finned tube bank (figure 1) are 
known. Once temperature evaluation for each finned tube layout is done, the inside fluid temperatures for each finned tube row are defined. The sensitivity analysis (section 4.1) of a finned tube bank shows that consideration applied for evaluating boundary conditions are satisfactory. These boundary conditions are employed in a numerical analysis of the finned tube bank showed in figure 1, which is described in section 2.3. Numerical predictions are compared with results from models for heat transfer and pressure drop in helically segmented finned tubes presented in section 2.2. The comparative analysis show close values between models and numerical results as discussed in results (section 4 ).

\subsection{Logarithmic Mean Temperature Difference (LMTD) method}

The methodology for calculating boundary conditions in the single isolated finned tube module is based on the Logarithmic Mean Temperature Difference method. This method allows the evaluation of heat transfer coefficients and friction factors for different geometries, according to models available in the open literature. These parameters permit the global evaluation of heat transfer and flow hydrodynamics of finned tube bank. The LMTD method considers the evaluation of overall heat transfer coefficient $(U)$, which is based on the outside finned tube and is defined with the following equation:

$$
U_{o}=\frac{1}{\frac{A_{o}+A_{o} R_{f o}\left(h_{o}+h_{r}\right)}{\left(h_{o}+h_{r}\right)\left(\eta_{f} A_{f}+A_{t}\right)}+\frac{e_{w} A_{o}}{k_{w} A_{i}}+\left(\frac{1}{h_{i}}+R_{f i}\right) \frac{A_{o}}{A_{i}}}
$$

where $h_{0}, h_{i}$, and $h_{r}$ are mean outside convective coefficient, mean inside convective coefficient, and radiation heat transfer coefficient, respectively. In the case of flue gas temperatures lower than $300^{\circ} \mathrm{C}$, the value of $h_{r}$ could be negligible [11], and so this value is considered zero. $R_{f o}$ and $R_{f}$, are the outside and inside fouling factors, respectively. $\eta_{f}, A_{f}, A_{t}$, $A_{o}$, and $A_{i}$ are fin efficiency, fin surface area, bare tube surface area, total surface area, and inside surface area, respectively. Finally, $e_{w}$ and $k_{w}$ are tube wall thickness and tube material thermal conductivity, respectively.

The mean convective coefficients are calculated for the inside and outside of finned tubes. The mean inside convective coefficient $\left(h_{i}\right)$ considered in the evaluation of $U$ is the Gnielinski's correlation [12]. This model has been validated with satisfactory results by Rane, et al. [13] and according to Bejan [14] is the best available in the open literature. The Gnielinski's correlation [12] is valid for $0.5 \leq \operatorname{Pr} \leq 2000$ and $3000 \leq \operatorname{Re} \leq 5 \times 10^{6}$, which is shown in the following equation:

$$
N u=\frac{h_{i} d_{i}}{k}=\frac{\left(f_{i} / 8\right)(\operatorname{Re}-1000) \operatorname{Pr}}{1+12.7(f / 8)^{1 / 2}\left(\operatorname{Pr}^{2 / 3}-1\right)}
$$

where $d_{i}$ and $k$ are inside diameter of tube and thermal conductivity of fluid. Re and Pr are the Reynolds Number and Prandtl Number. Finally, $f_{i}$ is the friction factor, which is defined in the following equation: 


$$
f_{i}=\frac{1}{\left(1.82 \log _{10} \operatorname{Re}-1.64\right)^{2}}
$$

There are many mean outside convective heat transfer coefficients $\left(h_{0}\right)$ in the open literature such as models proposed by Weierman [15], ESCOA [16], Nir [17], and Kawaguchi et al. [18]. The models attributable to Weierman [15] and Kawaguchi et al. [18] are recommended by Martinez et al. [19], but Kawaguchi's et al. [18] model is adopted. The model of Kawaguchi, et al. [18] is valid for $7000 \leq R e_{v} \leq 50000$ and $0.112 \leq s_{f} / d_{v} \leq 0.198$, which is shown in the next equation:

$$
N u=A_{2} \operatorname{Re}_{v}^{0.784} \operatorname{Pr}^{1 / 3}\left(s_{f} / d_{v}\right)^{-0.062}=\frac{h_{o} d_{v}}{k_{g}}
$$

where $R e_{v}$ is the Reynolds number based on the volume-equivalent diameter. The terms $A_{2}$, $S f$, and $d_{v}$ are the experimental coefficients for tube rows, fin gap, and volume-equivalent diameter, respectively. The coefficient $A_{2}$ is obtained from Kawaguchi's, et al. [18] model. Finally, $\mathrm{kg}$ is the thermal conductivity of gases.

The volume-equivalent diameter, $d_{v}$, is defined by the following equation:

$$
d_{v}=\left[t_{f} n_{f}\left\{\left(d_{o}+2 l_{f}\right)^{2}-d_{o}^{2}\right\}+d_{o}^{2}\right]^{1 / 2}
$$

where $n_{f}, t_{f}, l_{f}$, and $d_{o}$ are fin number per unit length, fin thickness, fin height, and outside diameter of bare tube, respectively.

Gas phase pressure drop can be calculated with models proposed by Weierman [15], ESCOA [16], Nir [17], and Kawaguchi et al. [18]. The model of Weierman [15] has been validated with satisfactory results by Martinez, et al. [19] and as a consequence, is adopted. In the analysis of compact heat recoveries a maximum pressure drop of $248.9 \mathrm{~Pa}$ [20] (1 inch (in) of water column (wc)) is considered in order to avoid technical problems such as backpressure. The pressure drop is calculated with the following empirical equation:

$$
\Delta P_{g}=\frac{\left(f_{o}+\frac{\left(1+B^{2}\right) \rho_{g p}}{4 N_{r}}\right) G_{o}^{2} N_{r}}{1.083 \times 10^{9} \rho_{g p}}
$$

where $B, G_{0}, N_{r}, f_{o}$, and $\rho_{g p}$ are the contraction factor, the gas mass flux, the number of tube rows, the friction factor, and the gas-phase density at the average outside temperature, respectively.

The gas phase friction factor $\left(f_{o}\right)$ is calculated with Weierman's model [15], which is valid for tube diameters between 38.1-60.96 $\mathrm{mm}$ and mass velocity of the gas between $0.67-40.36$ $\mathrm{kg} / \mathrm{m}^{2} \mathrm{~s}$. The Weierman's model [15] is shown in the following equation: 


$$
\begin{aligned}
& f_{o}=\left[0.07+8 \operatorname{Re}_{o}^{-0.45}\right] 0.11\left(\frac{0.05 S_{t}}{d_{o}}\right)^{-0.7\left(\frac{l_{f}}{s_{f}}\right)^{0.23}} \\
& {\left[1.1+\left(1.8-2.1 e^{-0.15 N_{r}^{2}}\right) e^{-\frac{2 S_{l}}{S_{t}}}-\left(0.7-0.8 e^{-0.15 N_{r}^{2}}\right) e^{-\frac{0.6 S_{l}}{S_{t}}}\right]\left(\frac{d_{f}}{d_{o}}\right)^{1 / 2}}
\end{aligned}
$$

where $R e_{o}$ is the Reynolds number based on the outside bare and $c_{p}$ is the specific heat capacity at constant pressure. Also, $d_{f}$ and $d_{0}$ are the outside diameter of the finned tube and the outside diameter of the bare tube, respectively. The terms $S_{l}$ and $S_{t}$ indicate the longitudinal pitch and the transverse pitch, respectively.

For more details about thermal evaluation of helically segmented finned tube banks see reference [21].

\subsection{Sensitive analysis of finned tube bank}

The methodology is based on the equivalence of a finned tube bank with single isolated finned tubes modules layouts. This methodology, which is based on the LMTD method, requires that predictions of friction factor and Nusselt number be independent of finned tube layout and number of finned tubes involved in compact heat recoveries. Then, a sensitive analysis of the finned tube bank is required in order to validate considerations in the methodology. The sensitive analysis is done for a small heat recovery (figure 1), in which results of friction factor and Nusselt number are compared for different finned tube layouts and finned tube lengths. The geometric characteristics of helically segmented finned tube and finned tube layout are shown in figure 3 . In this figure, a front and side views of the finned tube used in this study and its staggered configuration is presented. The finned tube bank analyzed corresponds to the layout shown in figure 1, which is composed by 4 finned tubes per row and 6 finned tube rows. The small heat recovery (figure 1) is analyzed with LMTD method for different finned tube configurations.

The finned tube configurations used in the sensitive analysis considers an initial finned tube layout of 2 finned tubes per row and 2 finned tubes rows (red polygon in figure 4). Subsequently, one finned tube row is added to reach 6 finned tube rows (red dash boxes in figure 4). Then, another finned tube is added to the row (blue polygon, figure 4) in order to obtain 3 finned tubes per row and 2 finned tube rows. Previous procedures in initial finned tube layout are done until 6 finned tube rows (blue dash boxes, figure 4) are reached. Finally, one last finned tube row is added (green polygon in figure 4 ) in order to get 4 finned tubes per row and 2 finned tube rows. The same procedure for previous finned tube layouts are done until full finned tube bank (gray dashed box) is reached. Once finned tube bank layout analysis is done, a similar study is proposed for different finned tube length. The procedure is the same as previous analysis but at different finned tube length. The finned tube lengths proposed are $1 \mathrm{~m}, 0.5 \mathrm{~m}$, and $0.05194 \mathrm{~m}$. The last 
finned tube length corresponds to the value used in the numerical simulation. The results (section 4.1) show variations lower than 1\% for Nusselt number and $3.6 \%$ lower for friction factor. These results confirm that considerations employed in this methodology (boundary condition calculation) are appropriate. The analysis is obtained with the thermodynamic conditions presented in table 1 for different finned tubes per row and finned tubes length which, shows deviations on Reynolds number based in the outside diameter tube $\left(\mathrm{Re}_{\mathrm{o}}\right)$ lower than $0.4 \%$. The variations in $\mathrm{Re}_{o}$ are due to adjustment of mass flow at different finned tube configurations.
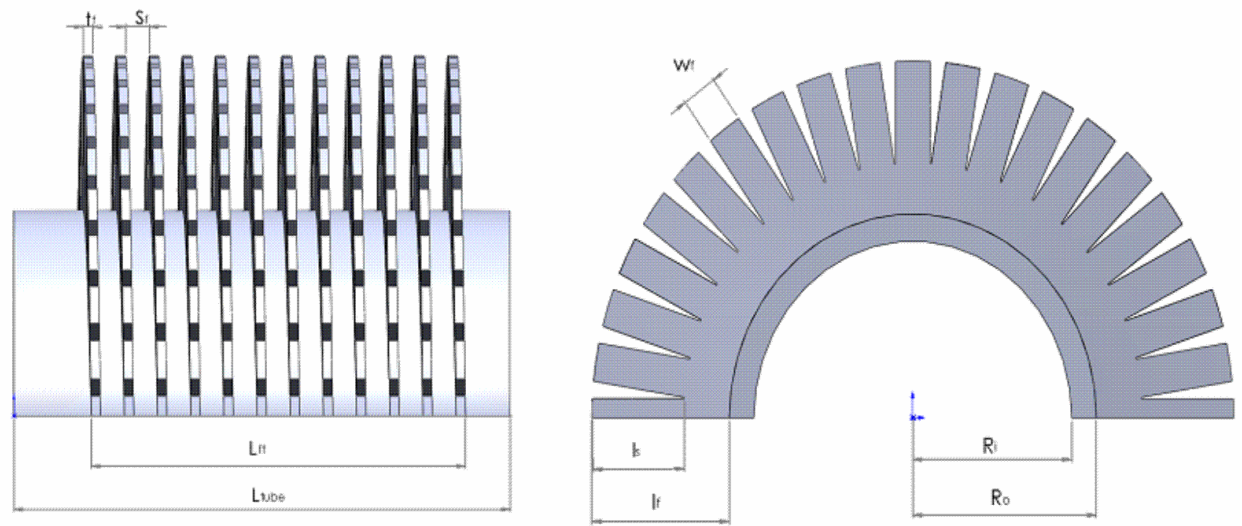

\begin{tabular}{|c|c|c|c|c|c|}
\hline \multicolumn{2}{|c|}{ Tube } & \multicolumn{2}{|c|}{ Fins } & \multicolumn{2}{|c|}{ Finned tube lavout } \\
\hline$D_{0}(m)$ & 0.0508 & $1_{\mathrm{f}}(\mathrm{m})$ & 0.01905 & $\mathrm{~S}_{\mathrm{T}}(\mathrm{m})$ & 0.11430 \\
\hline $\mathrm{D}_{\mathrm{i}}(\mathrm{m})$ & 0.044 & $1_{s}(\mathrm{~m})$ & 0.01275 & $\mathrm{~S}_{\mathrm{L}}(\mathrm{m})$ & 0.09906 \\
\hline $\mathrm{L}_{\mathrm{tf}}(\mathrm{m})$ & 1.0 & $t_{f}(m)$ & 0.0012 & $\theta\left({ }^{\circ}\right)$ & 60 \\
\hline $\mathrm{L}_{\mathrm{b}}(\mathrm{m})$ & 0 & $\mathrm{~S}_{\mathrm{f}}(\mathrm{m})$ & 0.003 & tube rows & 6 \\
\hline $\mathrm{L}_{\text {tube }}(\mathrm{m})$ & 1.0 & $\mathrm{w}_{\mathrm{f}}(\mathrm{m})$ & 0.0048 & tube per row & 4 \\
\hline & & $\mathrm{d}_{\mathrm{f}}($ fins $/ \mathrm{m})$ & 236.0 & & \\
\hline
\end{tabular}

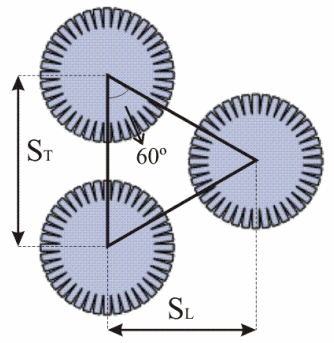

Figure 3. Geometric characteristics of finned tubes and finned tube layout. 


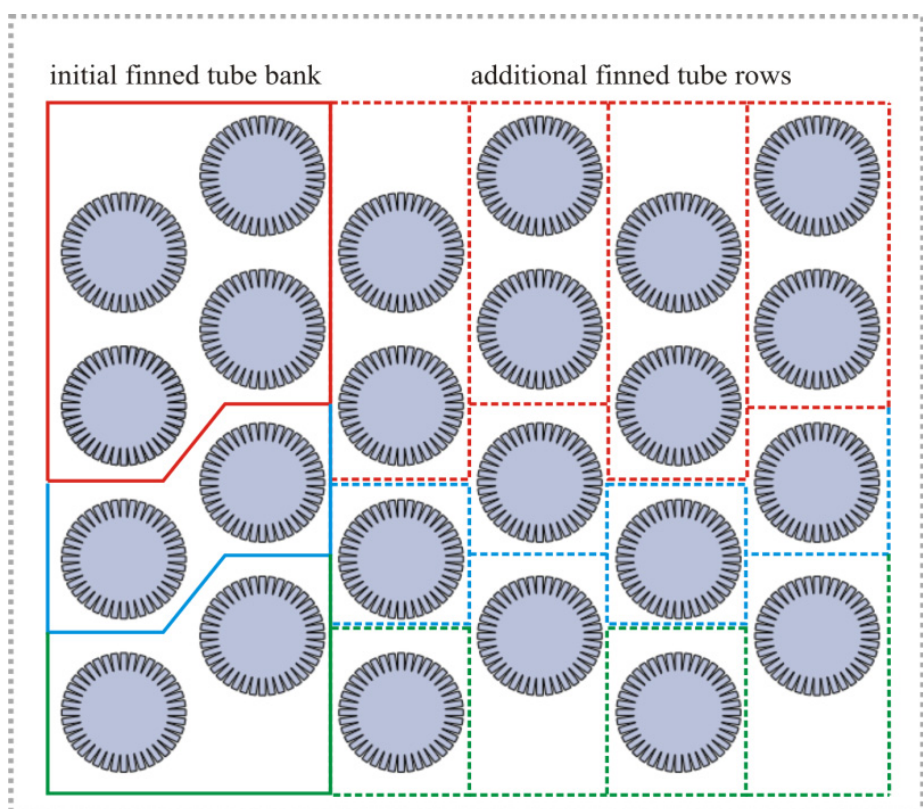

complete finned tube bank

Figure 4. Finned tube layout configurations in sensitive analysis.

\begin{tabular}{|c|c|c|c|}
\hline \multicolumn{4}{|c|}{ Finned tube length: $1 \mathrm{~m}$} \\
\hline & \multicolumn{3}{|c|}{ Finned tubes per row } \\
\hline Parameter & 2 & 3 & 4 \\
\hline mass flow $(\mathrm{kg} / \mathrm{s})$ & 0.3624 & 0.05436 & 0.7248 \\
\hline $\mathrm{Re}_{\circ}$ & 8562 & 8562 & 8562 \\
\hline $\mathrm{Ma}$ & $<0.03$ & $<0.03$ & $<0.03$ \\
\hline Temperature $\left({ }^{\circ} \mathrm{C}\right)$ & 60 & 60 & 60 \\
\hline \multicolumn{4}{|c|}{ Finned tube length: $0.5 \mathrm{~m}$} \\
\hline & \multicolumn{3}{|c|}{ Finned tubes per row } \\
\hline Parameter & 2 & 3 & 4 \\
\hline mass flow $(\mathrm{kg} / \mathrm{s})$ & 0.1812 & 0.2718 & 0.3624 \\
\hline $\mathrm{Re}_{\circ}$ & 8567 & 8567 & 8567 \\
\hline Мa & $<0.03$ & $<0.03$ & $<0.03$ \\
\hline Temperature $\left({ }^{\circ} \mathrm{C}\right)$ & 60 & 60 & 60 \\
\hline \multicolumn{4}{|c|}{ Finned tube length: $0.05194 \mathrm{~m}$} \\
\hline & \multicolumn{3}{|c|}{ Finned tubes per row } \\
\hline Parameter & 2 & 3 & 4 \\
\hline mass flow $(\mathrm{kg} / \mathrm{s})$ & $9.411 \times 10^{-3}$ & $14.116 \times 10^{-3}$ & $18.822 \times 10^{-3}$ \\
\hline $\operatorname{Re}_{\circ}$ & 8599 & 8599 & 8599 \\
\hline $\mathrm{Ma}$ & $<0.03$ & $<0.03$ & $<0.03$ \\
\hline Temperature $\left({ }^{\circ} \mathrm{C}\right)$ & 60 & 60 & 60 \\
\hline
\end{tabular}

Table 1. Thermodynamic conditions. 


\section{Numerical simulation}

In this section, the methodology for calculating boundary conditions in a single isolated finned tube module is employed in a numerical simulation of helically segmented finned tube bank in staggered layout. The objective is to apply the methodology in a numerical model that represents the interaction between the hydrodynamics and the heat transfer of a turbulent gas flow in complex systems. The simulation allows detailed analysis on compact heat recoveries that can be used for improving thermal behaviour. So, a correct implementation of boundary conditions in order to represent adequately physical phenomena is essential. The numerical model is focused to the outside finned tube, because the gas phase dominates the heat transfer [22] and also the pressure drop is critical [22]. However, the effect of the internal fluid is considered by means of an average inside temperature in each finned tube. Thus, the boundary conditions for outside flow and inside fluid are calculated with methodology described in section 2. In this numerical simulation, periodic boundary conditions are proposed for the single isolated finned tube module in the fully developed flow (Figure 1). Numerical predictions of Nusselt number and friction factor are compared with results obtained from correlations of Kawaguchi et al. [18] and Weierman [15], respectively in order to verify numerical results that depend on boundary conditions values.

\subsection{Governing equations}

The analysis of turbulent flows is complex, because fluid properties are irregular in space and time. Instantaneous variables $(\phi)$ are represented as a function of two terms: the mean $(\tilde{\varphi})$ and its fluctuation $\left(\varphi^{\prime \prime}\right)$. These terms are used in the transport equations that govern the flow movement and the heat exchange. The transport equations are averaged by means of Favre [23] method, which are solved for mean values of the fluid properties. Thereby, the set of mass, momentum, and energy transport equations in Cartesian system is defined as follows:

$$
\begin{gathered}
\frac{\partial \bar{\rho}}{\partial t}+\nabla \cdot(\bar{\rho} \tilde{\vec{V}})=0 \\
\frac{\partial}{\partial t}(\bar{\rho} \tilde{\vec{V}})+\nabla \cdot(\bar{\rho} \tilde{\vec{V}} \otimes \tilde{\vec{V}})=-\nabla \cdot \bar{P}+\mu \nabla^{2} \tilde{\vec{V}}-\nabla \cdot \overline{\left(\bar{\rho} \vec{V}^{\prime \prime} \vec{V}^{\prime \prime}\right)}+\overline{\rho \vec{g}} \\
\frac{\partial}{\partial t}(\bar{\rho} \tilde{h})+\nabla \cdot(\bar{\rho} \tilde{\vec{V}} \tilde{h})=-\nabla \cdot \tilde{\vec{J}}_{h}-\nabla \cdot \overline{\left(\bar{\rho} \vec{V}^{\prime \prime} h^{\prime \prime}\right)}
\end{gathered}
$$

where $\bar{\rho}, \tilde{\vec{V}}, \vec{V}^{\prime \prime}$, and $\tilde{h}$ are the mean density, instantaneous velocity, fluctuating velocity, and mean enthalpy, respectively. $\mu$ and $\overline{\vec{g}}$ are the viscosity and the gravitational acceleration, respectively. The terms $\overrightarrow{\bar{\rho} \vec{V}^{\prime \prime} \vec{V}^{\prime \prime}}$ and $\overrightarrow{\bar{\rho} \vec{V}^{\prime \prime} h^{\prime \prime}}$ are the apparent Reynolds stress tensor and the turbulent heat flux, respectively. $\overline{\bar{P}}$ is the pressure tensor. Finally, $\nabla \cdot \tilde{\vec{J}}_{h}$ is the diffusive heat flux, which is modeled with Fourier's law. 
Equations (2) and (3) demand additional mathematical expressions to model $\widetilde{V^{\prime \prime} \varphi^{\prime \prime}}$ term. The closure of these equations requires modeling of Reynolds stress tensor and of the turbulent heat flux. Then, the Reynolds stress tensor is closed with turbulence models. In this work, the k- $\varepsilon$ RNG (Renormalization Group) turbulence model developed by Yakhot and Orzag [24] is considered. The turbulent heat flux vector is obtained by means of an analogy between momentum transfer and thermal energy transfer. Under this concept, it is possible to establish a suitable articulation between the turbulent heat flux vector and the turbulent flow viscosity.

\subsection{Boundary conditions}

The implementation of periodic boundary conditions alleviates the computational resources, because the computational domain is reduced considerably. So, only a single isolated finned tube module in the fully developed flow region needs to be simulated (figure 1). Also, the tube length required (spatial direction y in figure 1) is minimum because only is necessary the length for the flow does not vary in this spatial direction because periodic conditions assume no influence of walls or position in any direction. Then, the computational domain is reduced $99 \%$, as shown in table 2 , because only is necessary the single isolated finned tube module (figure 1) with a tube length of $0.05194 \mathrm{~m}$. However, the numerical simulation requires a correct inclusion of boundary conditions in order to adequately represent the physical phenomenon. So, the boundary conditions are applied to a turbulent air flow in a stationary and fully-developed flow regime. The methodology developed by Patankar et al. [25] and Kelkar and Patankar [26] is implemented in this work. This methodology was generated for laminar flows and as a consequence, only velocity, pressure and temperature are considered as a periodic behaviour. However, additional considerations for turbulent flows need to be implemented. These considerations should depend on the turbulence model selected.

The periodic velocity is based on the non slip condition on the boundary walls of the computational domain and negligible value of the velocity variation in every spatial direction. So, the analysis is presented for the flow direction $(z)$, which can be generalized for the remaining spatial directions. Thereby, the periodic condition for the component velocity in the flow direction $(\tilde{w})$ is defined as follows:

$$
\frac{\partial \tilde{w}}{\partial z}=0 \quad \tilde{u}=0 \quad \tilde{v}=0
$$

where $\tilde{u}, \tilde{v}, \tilde{w}$ are the mean velocity components in the respective spatial directions $x, y, z$.

In the case of fully developed flows, the velocity is the same at a characteristic length $(L)$, and this is shown in the next equation:

$$
\tilde{w}(x, y, z)=\tilde{w}(x, y, z+L)
$$

The periodic boundary velocities need an initial value $w_{b}$ (bulk velocity), which is calculated with the following equation: 


$$
w_{b}=\frac{1}{A_{y z}} \int_{0}^{x} \int_{0}^{y} w_{m e d} d x d y
$$

where $w_{m e d}$ is an initial velocity profile.

The pressure field is obtained from a periodic behavior taking into consideration a pressure drop in the flow direction. This implies that the pressure is defined by the sum of a periodic pressure term in the flow direction and an average pressure drop. This is represented in the following equation:

$$
P(x, y, z)=\tilde{P}(x, y, z)-\beta z
$$

where $\tilde{P}$ and $\beta$ are periodic pressure and average pressure-gradient in the flow direction.

\begin{tabular}{|l|c|c|c|c|}
\hline & \multicolumn{3}{|c|}{ Dimensions: } & \\
\hline Layout & $\mathrm{x}(\mathrm{m})$ & $\mathrm{y}(\mathrm{m})$ & $\mathrm{z}(\mathrm{m})$ & cells \\
\hline Finned tube bank & 0.4572 & 1.0 & 0.59878 & 34200000 \\
\hline Single isolated finned tube module & 0.1143 & 0.05194 & 0.20312 & 178512 \\
\hline \multicolumn{4}{|l}{} & \\
\hline \multicolumn{4}{|l|}{ Computational domain reduction: } & \\
\hline Finned tube bank & reference & & & \\
\hline Single isolated finned tube module & 99.6 & & & \\
\hline
\end{tabular}

Table 2. Finned tube layout dimensions.

These two terms are defined in the pair of equations (15) and (16):

$$
\begin{gathered}
\tilde{P}(x, y, z)=\tilde{P}(x, y, z+L) \\
\beta=\frac{\tilde{P}(x, y, z)-\tilde{P}(x, y, z+L)}{L}=\frac{\Delta \tilde{P}}{L}
\end{gathered}
$$

where $\Delta \tilde{P}$ is the pressure drop over a finned tubes module, which can be calculated through the empirical equation (6).

The temperature field is obtained with constant wall heat flux boundary condition, which considers a constant variation of temperature in the flow direction; that is, the heat transfer magnitude is the same from one finned tube module to another finned tube module. This boundary condition can be appropriate for the present study because it can be applied to turbulent flows. However, it is appropriate if a uniform heat transfer is found in the small finned tube bank simulation. The numerical results in small finned tube bank show a quasiconstant mean temperature (plane xy) in the flow direction as shown in results section.

The temperature field is obtained from a periodic behavior and an adjustment term of the temperature in the flow direction. Thus, the temperature is defined by a periodic temperature term in the flow direction and an average temperature adjustment term expressed in the next expression: 


$$
T(x, y, z)=\tilde{T}(x, y, z+L)+\gamma z
$$

where $\tilde{T}(x, y, z)$ and $\gamma$ are the mean temperature field and the temperature-gradient term, respectively. The temperature-gradient term can be calculated with the following equation:

$$
\gamma=\frac{\tilde{T}(x, y, z)-\tilde{T}(x, y, z+L)}{L}=\frac{\dot{Q}}{\dot{m}_{g} c_{p} L}
$$

where $\dot{Q}, \dot{m}$, and $c_{p}$ are heat addition, mass flow, and specific heat at constant pressure, respectively.

The heat addition in the flow direction for the single isolated module is determined with the next equation:

$$
\dot{Q}=U_{o} A_{T} \Delta T_{M L}
$$

where $U_{o}$ is the overall heat transfer coefficient, $A_{T}$ the overall finned surface and $\Delta T_{M L}$ the logarithmic mean temperature difference.

The value of $U_{o}$ is obtained from equation (1) and $A_{T}$ is obtained with geometry of finned tube. For more details about calculations of these parameters see reference [21].

The numerical simulation of turbulent flow on a single isolated module needs the implementation of periodic conditions for additional variables, according to the turbulence model. In the case of k- $\varepsilon$ RNG turbulence model, the turbulent kinetic energy $(k)$ and its dissipation rate $(\varepsilon)$ show a periodic behavior, according to Martínez [27]. So, the periodic condition for the turbulent kinetic energy and its dissipation rate are defined as follows:

$$
\begin{aligned}
& \tilde{k}(x, y, z)=\tilde{k}(x, y, z+L) \\
& \tilde{\varepsilon}(x, y, z)=\tilde{\varepsilon}(x, y, z+L)
\end{aligned}
$$

The inclusion of periodic boundary conditions in the simulation of single isolated module requires light changes in the governing equations. The mass conservation equation does not change and is evaluated by means of equation (1). In the case of pressure field, the momentum conservation equation needs to include the adjust term of average pressuregradient in the flow direction. So, the equation (2) can be written as:

$$
\left.\frac{\partial}{\partial t}(\bar{\rho} \tilde{\vec{V}})+\nabla \cdot(\bar{\rho} \tilde{\vec{V}} \otimes \tilde{\vec{V}})=\beta-\nabla \cdot \bar{P}+\mu \nabla^{2} \tilde{\vec{V}}-\nabla \cdot \overline{\left(\bar{\rho} \vec{V}^{\prime \prime} \vec{V}^{\prime \prime}\right.}\right)+\bar{\rho} \overline{\vec{g}}
$$

The term $\beta$ is included in the CFD code PHOENICS 3.5.1 [28] by means of an additional source term in the momentum equation. On the other hand, the evaluation of temperature field requires that energy conservation equation must be adjusted in order to include the cooling of gas phase in the flow direction. So, the equation (3) can be written as:

$$
\frac{\partial}{\partial t}(\bar{\rho} \tilde{h})+\nabla \cdot(\bar{\rho} \tilde{\vec{V}} \tilde{h})+\nabla \cdot(\tilde{\vec{V}} \gamma)=-\nabla \cdot \tilde{\vec{J}}_{h}-\nabla \cdot \overline{\left(\bar{\rho} \vec{V}^{\prime \prime} h^{\prime \prime}\right)}
$$


The term $\nabla \cdot(\tilde{\vec{V}} \gamma)$ is included in the CFD code PHOENICS 3.5 .1 [28] by means of an additional source term in the energy equation.

The turbulent kinetic energy and the dissipation turbulent rate are calculated directly from equations (20) and (21) because these equations do not change.

\subsection{Numerical details}

The numerical simulation is developed for a single isolated finned tube module in the fully developed flow, which is shown in Figure 5. In this figure, the single isolated finned tube module is presented with boundary conditions calculated with methodology proposed. The dimensions of computational domain and the mesh used for simulation are shown in table 2. This table exhibits a reduction in computational domain with single isolated finned tube module of $99 \%$ that represents finite calculation times. The used mesh in numerical simulation considers that numerical predictions are independent from it. The thermodynamic employed conditions in the simulation work are presented in table 1 for finned tube length of $0.05194 \mathrm{~m}$ and figure 5. On the other hand, the complex geometry is represented by cut-cell method [29], which allows the use of Cartesian grids. The numerical simulations consider a staggered grid under a hybrid discretization scheme of the convective term. It was also considered that the system is in a stationary state and is only exposed to one gravitational field (in heat recoveries, the fluid generally flows in vertical direction), and that the gas discharge (finned tube bank outlet) occurs in a sea level atmosphere. Finally, the geometric characteristics of the finned tube used in this study are shown concurrently in Figure 3.

The numerical results are used for evaluating average Nusselt Number $(\mathrm{Nu})$ and average friction factor in small finned tube bank and single isolated module. The results are compared with values obtained from Correlations of Kawaguchi, et al [18] and Weierman [15] models, respectively.

The average Nusselt number is calculated with the next equation:

$$
\overline{N u}=\frac{\bar{h} d_{v}}{k_{g}}
$$

where $\bar{h}$ is the average convective coefficient, which is defined as:

$$
\bar{h}=\frac{\dot{Q}}{A_{o} \Delta T_{\text {LMnum }}}
$$

where $\Delta T_{\text {LMnum }}$ is the numerical-logarithmic mean temperature difference, which is calculated as:

$$
\Delta T_{\text {LMnum }}=\frac{\left[T_{i}(z+L)-T_{b}(z+L)\right]-\left[T_{i}(z)-T_{b}(z)\right]}{\operatorname{Ln} \frac{\left[T_{i}(z+L)-T_{b}(z+L)\right]}{\left[T_{i}(z)-T_{b}(z)\right]}}
$$




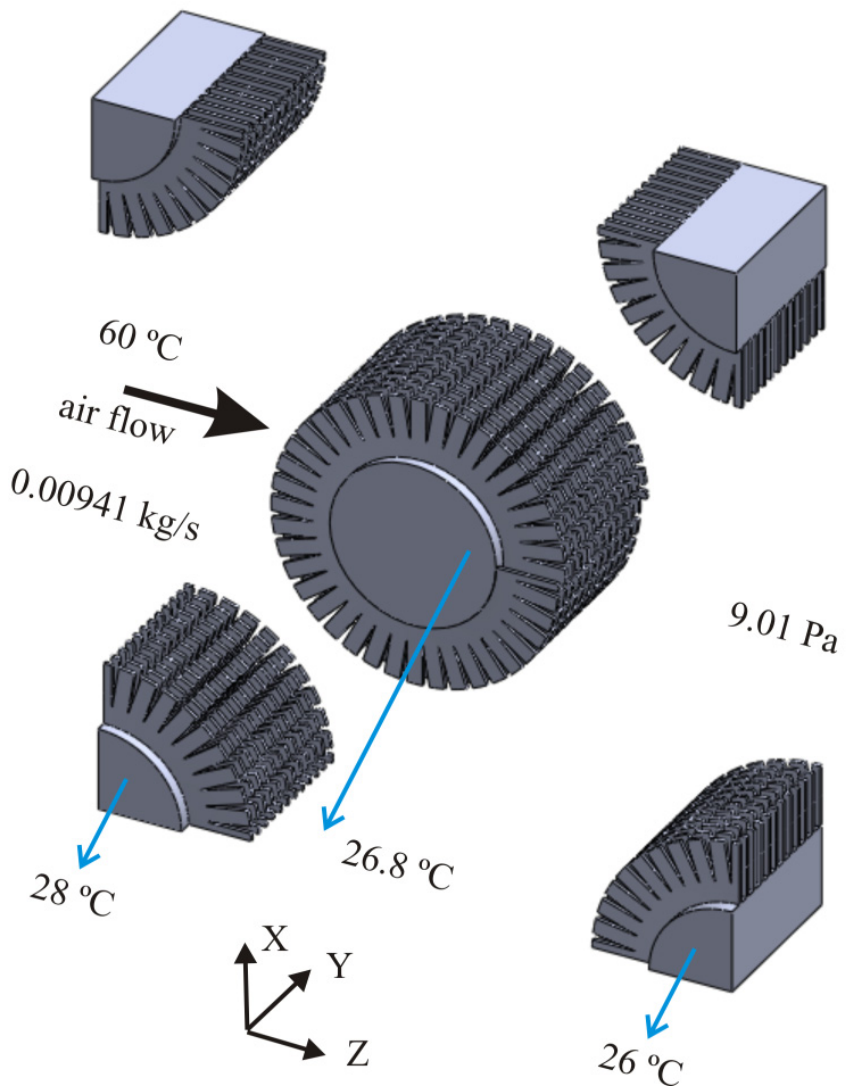

Figure 5. Computational domain in numerical simulation.

where $T_{i}$ and $T_{b}$ are the mean temperature inside finned tubes and mean boundary temperature, respectively. The conditions at $z$ and $z+L$ correspond to the inlet and exit of the single isolated finned tube module.

The numerical-average friction factor is obtained directly from equation (6) in which numerical pressure drop is calculated from the next expression:

$$
\Delta \tilde{P}=P_{b}(z)-P_{b}(z+L)
$$

where $P_{b}$ is the numerical-mean pressure.

\section{Results}

The methodology proposed is applied for evaluating boundary conditions in a single isolated finned tube module (figure 1). The predicted values are utilized in a numerical simulation in order to verify a correct representation of physical phenomena with these 
boundary conditions. These values require independence of results in heat transfer (Nusselt number) and pressure drop (friction factor) for different finned tube configurations, number of finned tubes, and finned tube lengths. So, a sensitive analysis is necessary in order to support previous considerations. The results of sensitive analysis shows that assumptions of methodology are correct, which are discussed in section 4.1. On the other hand, numerical predictions (focused in the outside flow) show a correct representation of interaction of heat transfer and flow hydrodynamics, which is presented in section 4.2. The comparative analysis of results between numerical predictions and results obtained from Kawaguchi et al [18] and Weierman [15] models for Nusselt number and friction factor, respectively, show close values as discussed in section 4.2.

\subsection{Sensitive analysis}

The LMTD method is applied to different finned tube layouts and finned tube lengths, which are described in section 2.3. The main goal of the analysis is to verify that friction factor and Nusselt number are independent of finned tube rows, number of finned tube per row, and finned tube length for the finned tube bank showed in figure 1 . The comparative analysis at different finned tube configurations and finned tube lengths with thermodynamic conditions presented in table 1 are shown in table 4 . In this table, Nusselt number and friction factor are presented at different finned tube configurations from 2 finned tubes per row and 2 finned tubes rows (red polygon in figure 4) until reach 4 finned tubes per row and 6 finned tubes rows (gray dashed box, figure 4). Evaluations are done for tube lengths of $1 \mathrm{~m}, 0.5 \mathrm{~m}$ y $0.05194 \mathrm{~m}$ as shown in table 3 . Predictions show constant values of Nusselt number and friction factor for the same finned tube rows. So, the Nusselt number varies from 100.3 to 99.3 and the friction factor changes from 0.31 to 0.321 for different finned tube rows ( 2 to 6 ) of the small heat recovery. These results exhibit independence of predictions in dimensionless parameters with finned tube length and finned tubes per row in a heat recovery. The only variation of results is for configurations at different finned tube rows as shown in table 3 . The main reason of dimensionless parameters variation is temperature reached by the gas phase (outside flow). These temperature variations slightly affect results because the gas phase is cooled in $3{ }^{\circ} \mathrm{C}$ temperature difference for configurations analyzed at different finned tube rows. The results exhibit variations lower than $1 \%$ for Nusselt number and $3.6 \%$ for friction factor. These deviations are calculated for different finned tube rows, which is not representative of the same physical phenomenon because different finned tube layouts represent different gas cooling. In spite of this situation, the results show close values in Nusselt number and friction factor. Therefore, assumptions considered in the methodology are correct because finned tube bank performance is independent of finned tube rows, number of finned tubes per row, and finned tube length in a finned tube bank. This methodology allows numerical studies in heat recoveries at industrial scale because the computational domain can be reduced in $99 \%$ as shown in table 2 . So, the calculation times are finites due to the analysis is focused in a single isolated finned tube module in the fully developed flow. 


\begin{tabular}{|c|c|c|c|c|c|c|}
\hline \multicolumn{7}{|c|}{ Finned tube length: $1 \mathrm{~m}$} \\
\hline & \multicolumn{6}{|c|}{ Finned tubes per row } \\
\hline & \multicolumn{2}{|c|}{2} & \multicolumn{2}{|c|}{3} & \multicolumn{2}{|c|}{4} \\
\hline Finned tube rows & $\mathrm{Nu}$ & $\mathrm{f}$ & $\mathrm{Nu}$ & $\mathrm{f}$ & $\mathrm{Nu}$ & $\mathrm{f}$ \\
\hline 2 & 99.3 & 0.321 & 99.3 & 0.321 & 99.3 & 0.321 \\
\hline 3 & 99.6 & 0.319 & 99.6 & 0.319 & 99.6 & 0.319 \\
\hline 4 & 99.8 & 0.313 & 99.8 & 0.313 & 99.8 & 0.313 \\
\hline 5 & 100.01 & 0.311 & 100.01 & 0.311 & 100.01 & 0.311 \\
\hline 6 & 100.3 & 0.31 & 100.3 & 0.31 & 100.3 & 0.31 \\
\hline \multicolumn{7}{|c|}{ Finned tube length: $0.5 \mathrm{~m}$} \\
\hline & \multicolumn{6}{|c|}{ Finned tubes per row } \\
\hline & \multicolumn{2}{|c|}{2} & \multicolumn{2}{|c|}{3} & \multicolumn{2}{|c|}{4} \\
\hline Finned tube rows & $\mathrm{Nu}$ & $\mathrm{f}$ & $\mathrm{Nu}$ & $\mathrm{f}$ & $\mathrm{Nu}$ & $\mathrm{f}$ \\
\hline 2 & 99.3 & 0.321 & 99.3 & 0.321 & 99.3 & 0.321 \\
\hline 3 & 99.6 & 0.319 & 99.6 & 0.319 & 99.6 & 0.319 \\
\hline 4 & 99.8 & 0.313 & 99.8 & 0.313 & 99.8 & 0.313 \\
\hline 5 & 100.01 & 0.311 & 100.01 & 0.311 & 100.01 & 0.311 \\
\hline 6 & 100.3 & 0.31 & 100.3 & 0.31 & 100.3 & 0.31 \\
\hline \multicolumn{7}{|c|}{ Finned tube length: $0.05194 \mathrm{~m}$} \\
\hline & \multicolumn{6}{|c|}{ Finned tubes per row } \\
\hline & \multicolumn{2}{|c|}{2} & \multicolumn{2}{|c|}{3} & \multicolumn{2}{|c|}{4} \\
\hline Finned tube rows & $\mathrm{Nu}$ & $\mathrm{f}$ & $\mathrm{Nu}$ & $\mathrm{f}$ & $\mathrm{Nu}$ & $\mathrm{f}$ \\
\hline 2 & 99.3 & 0.321 & 99.3 & 0.321 & 99.3 & 0.321 \\
\hline 3 & 99.6 & 0.319 & 99.6 & 0.319 & 99.6 & 0.319 \\
\hline 4 & 99.8 & 0.313 & 99.8 & 0.313 & 99.8 & 0.313 \\
\hline 5 & 100.01 & 0.311 & 100.01 & 0.311 & 100.01 & 0.311 \\
\hline 6 & 100.3 & 0.31 & 100.3 & 0.31 & 100.3 & 0.31 \\
\hline
\end{tabular}

Table 3. Sensitivity analysis results.

\subsection{Numerical results}

The predictions for velocity field, pressure field, and temperature field are shown in Figure 6. In all figures, profiles of the variables are presented on the $x-z$ plane, because this is the plane that exhibits most changes in properties. The velocity contours (figure 6a) reflect an apparently symmetric behaviour but the field is slight asymmetry due to the fin helical layout. The recirculation zones are observed at the rear portion of the tubes, with reference to air flow direction. These recirculation zones are narrow because the flow tends to stick to the contour of finned tubes by turbulence generated. The recirculation region has amplitude and length of approximately $49.85 \mathrm{~mm}$ and $52.61 \mathrm{~mm}$, respectively. The flow tends to accelerate in the free zones where the cross-section area is smaller (central region of computational domain and at the side portions of the central finned tube) with a maximum velocity of $5.017 \mathrm{~m} / \mathrm{s}$. Finally, the backwater area where the flow is stopped abruptly at the central portion of the finned tube exhibit has a width and a length of about $9.75 \mathrm{~mm}$ and $6.57 \mathrm{~mm}$, respectively. 
The pressure contours (figure 6b) indicate an apparently symmetric pressure profile, similar to the velocity profile, which is not symmetric due to the helical fin. The results show a highpressure zone at the front portion of the tube, taking the flow direction as the point of reference. This high-pressure zone is created due to flow stopping abruptly at the central portion of the finned tube, producing a backwater zone. At the rear of the finned tube, there is a low-pressure area that is stratified at the outlet of single isolated module. The mean pressure at the inlet and outlet of the module is $23.184 \mathrm{~Pa}$ and $9.019 \mathrm{~Pa}$, respectively, as shown in table 4. The pressure drop is $14.165 \mathrm{~Pa}$, which represents a friction factor of 0.311 (table 4). The deviation of numerical results is $3.13 \%$ and $0.32 \%$ for pressure drop and friction factor, respectively. So, numerical predictions with boundary conditions calculated from methodology correspond to results obtained with Weierman's [15] model.

Numerical predictions of temperature contours (figure 6c) show an apparently symmetric profile, which are not symmetric due to the helical fin. The results exhibit that the hightemperature region is located at the central region of the computational domain, whereas the low-temperature areas are located at the rear of the finned tubes, viewed with respect to the flow direction. So, the most important heat transfer effects occur at the front of the extended surfaces and at the sides of the finned tube between the backwater zone and the recirculation zone. This pattern is attributed to the air flow coming into abrupt contact with the finned tubes, and to turbulence being created at the sides of the finned tubes. On the other hand, the temperature contours exhibit that flow temperature is dominated for inside fluid temperature in the recirculation zone. The mean temperature at inlet and outlet of module is $43.76^{\circ} \mathrm{C}$ and $34.73^{\circ} \mathrm{C}$ (table 4). The temperature difference is $9.03^{\circ} \mathrm{C}$, which represents a numerical-mean Nusselt number of 95.1, as shown in table 4 . Numerical results show deviations of $4.25 \%$ and $5.18 \%$ for temperature difference and Nusselt number, respectively. These results show that numerical predictions are close to results obtained with Kawaguchi's et al. [18] model. So, the numerical simulation represents adequately heat transfer in a single isolated finned tube module with boundary conditions calculated from methodology.

\begin{tabular}{|c|c|c|}
\hline & Numerical Simulation & model \\
\hline & & Weierman \\
\hline$P_{\text {inlet }}(\mathrm{Pa})$ & 24.44 & na \\
\hline $\mathrm{P}_{\text {outlet }}(\mathrm{Pa})$ & 10.27 & na \\
\hline$\Delta \mathrm{P}(\mathrm{Pa})$ & 14.17 & 13.74 \\
\hline deviation & $3.13 \%$ & reference \\
\hline $\mathrm{f}$ & 0.311 & 0.31 \\
\hline deviation & $0.32 \%$ & reference \\
\hline & & Kawaguchi \\
\hline Tinlet $\left({ }^{\circ} \mathrm{C}\right)$ & 40.78 & na \\
\hline $\mathrm{T}_{\text {outlet }}\left({ }^{\circ} \mathrm{C}\right)$ & 31.78 & na \\
\hline$\Delta \mathrm{T}\left({ }^{\circ} \mathrm{C}\right)$ & 9.0 & 9.4 \\
\hline deviation & $4.25 \%$ & reference \\
\hline $\mathrm{Nu}$ & 95.1 & 100.3 \\
\hline deviation & $5.18 \%$ & reference \\
\hline
\end{tabular}

Table 4. Comparative results. 


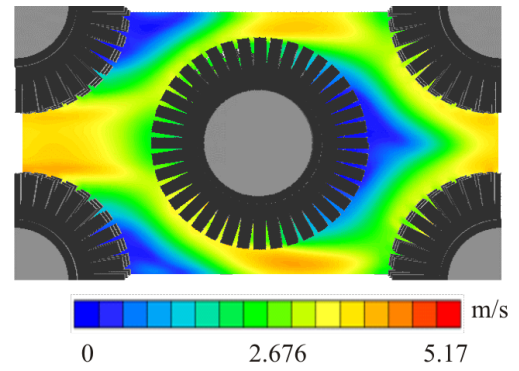

a) velocity contours

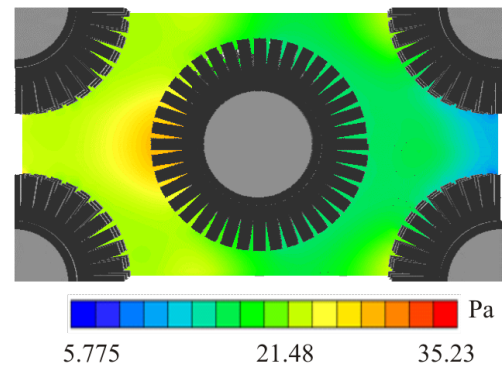

b) pressure contours

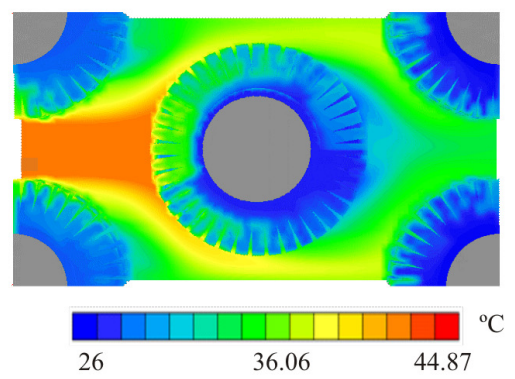

c) temperature contours

Figure 6. Numerical predictions.

\section{Conclusion}

The methodology, based on the LMTD method, for calculating boundary conditions in a single isolated finned tube module is adequate because the Nusselt number and friction factor show a quasi-constant behaviour for different finned tube length and finned tubes per row. The maximum deviations for Nusselt number and friction factor are lower than $1 \%$ and $3.6 \%$, respectively. Therefore, the methodology can be used in numerical analysis of heat recoveries at industrial scale because the computational domain can be reduced in $99 \%$ which allows finite computational times. Numerical predictions in the single isolated finned tube module show that pressure contours are adequate because mean pressure drop and mean friction factor exhibit a deviation of $3.13 \%$ and $0.32 \%$ with respect to model developed by Weierman [15]. On the other hand, the pressure contours exhibit that the high pressure values are located in backwater zone and the main dissipative effect of flow energy is located at this region. Therefore, the pressure drop is dominated for backwater zone. The temperature field show a deviation of $4.25 \%$ and $5.18 \%$ for temperature difference and Nusselt number with respect to Kawaguchi's, et al [18] model. The temperature contours exhibit that bare tube temperature is dominated by inside fluid temperature. Therefore, the inside fluid temperature must be considered as a lower limit temperature for cooling of flue gases in order to avoid dew point of acid gases. 


\section{Nomenclature}

\section{Symbols}

A

surface area

$A_{2}$

tube row coefficient

$B$

contraction factor

$c_{p}$

specific heat at constant pressure

d diameter

e thickness

f friction factor

G gas mass flux

$\overline{\vec{g}}$

gravitational acceleration

$\bar{h}$

average convective coefficient

$\tilde{h}$

mean enthalpy

$h$

mean convective coefficient

$k$

$\tilde{k}$

thermal conductivity

turbulent kinetic energy

$L \quad$ characteristic length

$l \quad$ height

$\dot{m}$ mass flow

$\mathrm{N} \quad$ Number of tubes

$n$ number

$\mathrm{Nu} \quad$ Nusselt Number

$\overline{N u} \quad$ average Nusselt number

$\tilde{P} \quad$ periodic pressure

$P_{b} \quad$ mean pressure

Pr Prandtl number

$\dot{Q} \quad$ heat addition

Re Reynolds number

$R_{f} \quad$ fouling factor

$S \quad$ pitch

$s \quad$ gap

$\tilde{T} \quad$ periodic temperature

$T$ temperature

t thickness

$U \quad$ overall heat transfer coefficient

$\tilde{u}, \tilde{v}, \tilde{w} \quad$ mean velocity components

$\tilde{\vec{V}} \quad$ instantaneous velocity

$\vec{V}^{\prime \prime} \quad$ fluctuating velocity

w velocity

$\Delta \tilde{P} \quad$ pressure drop 
$\triangle T_{M L} \quad$ logarithmic mean temperature difference.

$\begin{array}{ll}\text { Subscripts } & \\ \mathrm{b} & \text { bulk, boundary } \\ \mathrm{f} & \text { fin } \\ \mathrm{g} & \text { gases } \\ g p & \text { gas-phase } \\ \mathrm{i} & \text { inside } \\ \mathrm{l} & \text { longitudinal } \\ \mathrm{med} & \text { Initial profile } \\ \mathrm{num} & \text { numeric } \\ \mathrm{O} & \text { outside (tube diameter), overall } \\ \mathrm{r} & \text { Radiation, rows } \\ \mathrm{t} & \text { bare tube, transverse } \\ \mathrm{v} & \text { volume-equivalent diameter } \\ \mathrm{w} & \text { wall, tube material }\end{array}$

\section{Greek letters}

$\beta \quad$ average-pressure gradient

$\bar{\rho} \quad$ mean density

Q density

$\tilde{\varepsilon} \quad$ dissipation turbulent rate

$\eta \quad$ efficiency

$\mu \quad$ viscosity

$\gamma \quad$ temperature-gradient term

\section{Author details}

E. Martínez and G. Soto

Universidad Autónoma Metropolitana - Azcapotzalco, Mexico City, Mexico

W. Vicente and M. Salinas

Instituto de Ingeniería, Universidad Nacional Autónoma de México,

Ciudad Universitaria, Mexico City, Mexico

A. Campo

Department of Mechanical Engineering, University of Texas at San Antonio, San Antonio, TX, USA

\section{Acknowledgement}

We appreciate the support given to the research presented here by Universidad Nacional Autonoma de Mexico (Direccion General de Asuntos del Personal Academico, PAPIITIN106112-3), and Universidad Autonoma Metropolitana Azcapotzalco. 


\section{References}

[1] Beale, S. B, and Spalding, D. B. (1999). A Numerical Study of Unsteady Fluid Flow in Inline and Staggered Tube Banks. Journal of Fluids and Structures, Vol.13, No.6, pp. 723754, ISSN 0889-9746.

[2] Comini, G, and Croce, G. (2003). Numerical Simulation of Convective Heat and Mass Transfer in Banks of Tubes. International Journal for Numerical Methods in Engineering, Vol.57, No.12, pp. 1755-1773, ISSN 1755-1773.

[3] Beale, S. B. (2007). Use of Streamwise Periodic Boundary Condition for Problems in Heat and Mass Transfer. ASME Journal of Heat Transfer, Vol.129, No.4, pp. 601-605, ISSN 00221481.

[4] Beale, S. B. (2008). Benchmark Studies for the Generalized Streamwise Periodic Heat Transfer Problem. ASME Journal of Heat Transfer, Vol.130, No.11, pp. 114502_1-114502_4, ISSN 00221481.

[5] Benhamadouche, S, and Laurence, D. (2003). LES, Coarse LES, and Transient RANS Comparisons on the Flow Across a Tube Bundle. International Journal of Heat and Fluid Flow, Vol.24, No.4, pp. 470-479, ISSN 0142-727X.

[6] M. Salinas-Vázquez, M.A. de la Lama, W. Vicente, E. Martínez, (2011). Large Eddy Simulation of a Flow through Circular Tube Bundle. Applied Mathematical Modelling, Vol.35, No.9, pp. 4393-4406. ISSN 0307-904X.

[7] Hofmann, R, and Ponweiser, K. (2008). Experimental and Numerical Investigations of Serrated-Finned Tubes in Cross-Flow. Available from: www.zid.tuwien.ac.at/fileadmin/files_zid/projekte/2008/08-302-2.pdf.

[8] Mcilwain S. R. (2010). A Comparison of Heat Transfer Around a Single Serrated Finned Tube and a Plain Finned Tube. IJRAAS, Vol.2, No.2, pp. 88-94, ISSN

[9] Mcilwain S. R. (2010). A CFD Comparison of Heat Transfer and Pressure Drop Across Inline Arragement Serrated Finned Tube Heat Exchangers with an Increasing Number of Rows. IJRAAS, Vol.4, No.2, pp. 162-169, ISSN

[10] Lemouedda, A, Schmid, A, Franz, E, Breuer, M, Delgado, A. (2011). Numerical Investigations for the Optimization of Serrated Finned-Tube Heat Exchangers. Applied Thermal Engineering, Vol.31, No. 8-9, pp. 1393-1401, ISSN 1359-4311.

[11] Ganapathy, V. (2002). Industrial Boilers, Heat Recovery and Steam Generators: Design, Applications and Calculations. In Marcel Dekker, (Ed), 333-507, ISBN 0-8247-0814-8, New York.

[12] Gnielinski, V. (1976). New equations for heat and mass transfer in turbulent pipe and channel flow. Int. Chem. Eng. Vol.16, No.1, pp. 359-366, ISSN 0020-6318.

[13] Rane, M. V., Tandale, S. (2005). Water-to-water heat transfer in tube-tube heat exchanger: Experimental and analytical study. Applied Thermal Engineering, Vol.25, No.17, pp. 2715-2729, ISSN 1359-4311.

[14] Bejan, A. (1995). Convection Heat Transfer. In Wiley, (Ed), pp. 391-395, ISBN 0-47127150-0.

[15] Weierman, C. (1976). Correlations Ease The Selection of Finned Tubes. Oil and Gas Journal, Vol.74, No.36, pp. 94-100, ISSN 0030-1388. 
[16] ESCOA Turb-X HF Rating Instructions. (1979). Extended Surface Corporation of America (ESCOA), Pryor, OK.

[17] A. Nir. (1991). Heat Transfer and Friction Factor Correlations for Crossflow over Staggered Finned Tube Banks. Heat Transfer Engineering, Vol.12, No.1, pp. 43-58, ISSN 0145-7632.

[18] Kiyoshi Kawaguchi, Kenichi Okui, Takaharu Kashi. (2005). Heat transfer and pressure drop characteristics of finned tube banks in forced convection. Journal of Enhanced Heat Transfer, Vol.12, No.1, pp. 1-20, ISSN 1065-5131.

[19] E. Martinez, G. Soto, W. Vicente, M. Salinas. (2005). Comparative Analysis of Heat Transfer and Pressure Drop in Helically Segmented Finned Tube Heat Exchangers. Applied Thermal Engineering, Vol.30, No.11-12, pp. 1470-1476, ISSN 1359-4311.

[20] Jonh Weale, P.E., Peter H. Rumsey, P.E., Dale Sartor, P.E., and Lee Eng Lock. (2002). Laboratory Low-Pressure Drop Design. ASHRAE Journal, Vol. August pp. 38-42. ISSN 0001-2491.

[21] E. Martinez, W. Vicente, M. Salinas, G. Soto. (2010). Thermal Design Methodology of Industrial Compact Heat Recovery with Helically Segmented Finned Tubes. Heat Exchangers; design, types and applications. In Nova Publishers, (Ed), Series: Energy Science, Engineering and Technology, pp. 215-228. ISBN: 978-1-61761-308-1.

[22] Martínez, E, Vicente, W, Salinas, M, Soto, G. (2009). Single-phase experimental analysis of heat transfer in helically finned heat exchangers, Applied Thermal Engineering, Vol.29, No. 11-12, pp. 2205-2210, ISSN 1359-4311.

[23] Favre, A. (969). Problems of Hydrodynamics and Continuum Mechanics, SIAM.

[24] Yakhot, V, and Orszag, S. (1986). Renormalization Group Analysis of Turbulence. Basic Theory. Journal of Scientific Computing, Vol.1, No.1, pp. 3-51, ISSN 08857474.

[25] Patankar, S. V, Liu, C. H, Sparrow, E. M, 1977, Fully Developed Flow and Heat Transfer in Ducts Having Streamwise-Periodic Variations of Cross-Sectional Area, ASME Journal of Heat Transfer, 99, pp. 180-186.

[26] Kelkar, K. M, and Patankar, S. V, 1987, Numerical Prediction of Flow and Heat Transfer in a Parallel Plate Channel with Staggered Fins, ASME Journal of Heat Transfer, 109, pp. 25-30.

[27] Martínez, E. E. (2011). Simulación Numérica de un Flujo de Gases Turbulentos en un Banco de Tubos aletdos en Geometría Compleja. Ph.D Thesis. Universidad Autónoma Metropolitana, México City, México.

[28] J. C. Ludwing, H. Q. Qin, D. B. Spalding. (1989). The PHOENICS Reference Manual. Technical Report CHAM TR/200, CHAM Ltd, London.

[29] Y. Choi, J. Hong, H. Hwang, J. Choi. (2008). Cartesian Grid Method with Cut Cell in the Mold Filling Simulation. Journal Materials Science British Technology, Vol.24, No.3, pp. 379-382, ISSN 1005-0302. 